\section{CPS-044 THE ROUTINE USE OF ANTIBIOTICS AFTER INSERTION OF A CARDIAC IMPLANTABLE ELECTRONIC DEVICE (CIED): EVIDENCE AND CURRENT PRACTICE}

S Omran*, O Mukhtar, S Mousa. King Salman Armed Forces Hospital-Nwafh, Adult Intensive Care Unit, Tabuk, Saudi Arabia

\subsection{6/ejhpharm-2020-eahpconf.145}

Background and importance Cardiac implantable electronic devices (CIED) are used for patients with heart block and severe dysrhythmia to improve patient quality of life and survival. However, the implanted devices have been associated with an increased rate of infections and subsequently cause significant morbidity and mortality. Recent guidelines recommend the use of intravenous cefazolin as standard preoperative antibiotic prophylaxis. However, there is no consensus about postoperative antibiotic prescribing to treat infection. The routine practice in our clinical setting is to prescribe antibiotics pre and post insertion according to physician experience and preference. Hypothetically, if such practice continues, it may lead to an increased risk of antibiotic resistance, suboptimal clinical outcomes and higher healthcare costs.

Aim and objectives To investigate the rate of postoperative infection associated with CIED insertion, to find an association between prescribing antibiotics post insertion and postoperative infections and to develop recommendations that may help to optimise antimicrobial prescribing and minimise the consequences of infection and subsequently improve the practice.

Material and methods It this retrospective observational study, patient records (aged $\geq 18$ years old) with complete heart block who were admitted for permanent pacemaker (PPM) insertion were reviewed during the period January 2012 to December 2017. Patient demographic, comorbidities and microbiological reports through screening of blood culture within 90 days of post pacemaker insertion were collected. To find an association between antibiotic post insertion and postoperative infections, the $\chi^{2}$ or Fisher's exact test was applied. A p value $\leq 0.05$ was considered statistically significant.

Results Of 130 implanted device cases, 95 were reported as pacemaker (PPM) insertion cases during the study period; 67 (70\%) PPM cases were given post insertion antibiotics. No postoperative case of pocket infection or infective endocarditis was reported. Of 5 bacteraemia infections, only 1 case $(3 \%)$ was reported among the group who was not given antibiotics post insertion $(p=0.63)$.

Conclusion and relevance Antibiotic administration post pacemaker insertion has no added value in terms of infection prevention, and no evidence to support the use of antibiotics post pacemaker insertion. Therefore, this practice is not justified.

\section{REFERENCES AND/OR ACKNOWLEDGEMENTS}

No conflict of interest.

\section{CPS-045 COST EFFECTIVENESS ANALYSIS OF MEROPENEM DOSE OPTIMISATION IN CRITICAL PATIENTS}

Al Idoate*, A Aldaz, I Aquerreta, A Ortega. Clinica Universidad De Navarra, Pharmacy, Pamplona, Spain

10.1136/ejhpharm-2020-eahpconf.146
Background and importance Meropenem dose adjustment following pharmacokinetic/pharmacodynamic monitoring (TDM) in critical patients (CP) presents a clinical benefit. An economic analysis of this activity could facilitate its use in clinical practice. Aim and objectives To conduct a cost effectiveness analysis of meropenem TDM in CP versus standard dose (SD) according to the package insert recommendations.

Material and methods We conducted a naturalistic, retrospective, observational cohort study of $\mathrm{CP}$ receiving meropenem between May 2011 and December 2017 in a university hospital. Two cohorts were analysed: patients with meropenem TDM (cohort A) and patients with SD meropenem (cohort B).

The main effectiveness variable was the percentage of patients with a reduction of at least $80 \%$ in the procalcitonin value at the end of meropenem treatment compared with the maximum value during meropenem treatment.

Costs included in the analysis were: meropenem, material for drug preparation, TDM, time for preparation, administration and infusion surveillance, meropenem adverse drug reactions (ADR), critical care hospitalisation days and re-entries.

Propensity score (PS) matching was applied for patient selection. The $\chi^{2}$ was used to compare effectiveness and bootstrap to calculate the difference in costs between cohorts. A cost effectiveness analysis with deterministic and probabilistic sensitivity analyses was performed.

Results A total of 154 patients were included (77 per cohort) after PS matching. Meropenem dose was changed in 51 $(66.2 \%)$ patients with TDM, in most $(90.2 \%)$ because they were overdosed. In cohort A, 71.4\% of patients had reduced procalcitonin by at least $80 \%$ compared with $53.2 \%$ in cohort B (difference $18.2 \%$ (95\% CI 3.1; 33.2; p=0.020)). No significant differences were found in ADR between the two cohorts. An average decrease in cost per patient of $-1454 €$ (95\% CI $-4627 ; 1720 €$ ) with TDM was observed, with lower cost per patient for meropenem $-62 €(95 \%$ CI $-116 ;-4)$, disposable material $-12 €(95 \% \mathrm{CI}-29 ; 4)$ and nursing time $-38 €(95 \%$ CI $-71 ;-4)$ in cohort A, that offset the TDM cost $(47 €)$. Mean hospitalisation cost in patients with TDM was $8912 €$ versus $10325 €$ in cohort $B$. There was a $75 \%$ probability that TDM was more effective and cheaper (dominant) than SD according to the sensitivity analysis.

Conclusion and relevance Meropenem dose adjustment following pharmacokinetic/pharmacodynamic criteria was more effective, with similar safety and lower costs, than dosing according to the package insert recommendations.

\section{REFERENCES AND/OR ACKNOWLEDGEMENTS}

1. Schuetz $P$, et al. Procalcitonin-guided antibiotic stewardship. Clin Chem Lab Med 2019

No conflict of interest.

\section{CPS-046 MONITORING THE PRESCRIPTION OF NEW ANTIBIOTICS: THE WORK OF THE ANTIMICROBIAL STEWARDSHIP TEAM IN A THIRD LEVEL HOSPITAL}

E Sánchez-Yáñez, M Gómez Delgado, JL Ortiz Latorre*, MR Mora-Santiago, I MoyaCarmona. Hospital Universitario Virgen De La Victoria, Servicio De Farmacia, Málaga, Spain

\subsection{6/ejhpharm-2020-eahpconf.147}

Background and importance The prescriptions of new antibiotics should be done with caution as improper use can lead to the emergence of new antimicrobial resistance. The antimicrobial stewardship team (AST) and the commission of infections 